MEMORANDUM

RM-5192-NASA

APRIL 1967

\section{NATURE OF SURFACE FLOW IN THE EARTH'S CENTRAL CORE}

E. H. Vestine, R. H. Ball and Anne B. Kahle
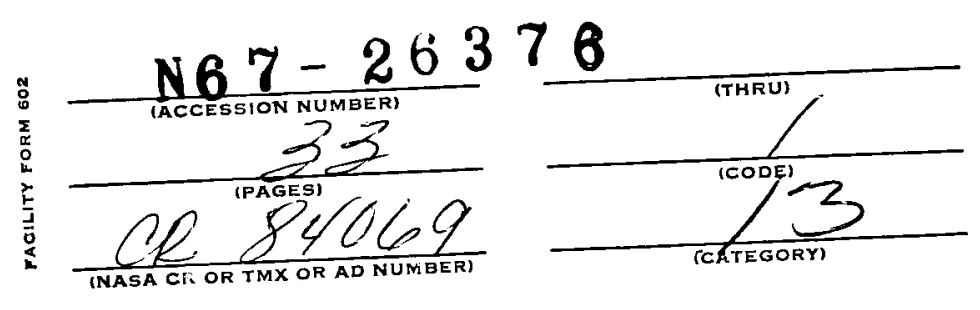

PREPARED FOR:

NATIONAL AERONAUTICS AND SPACE ADMINISTRATION 


\section{MEMORANDUM}

RM-5192-NASA

APRIL 1967

\section{NATURE OF SURFACE FLOW IN THE EARTH'S CENTRAL CORE \\ E. H. Vestine, R. H. Ball and Anne B. Kahle}

This research is sponsored by the National Aeronautics and Space Administration under Contract No. NASr-21. This report does not necessarily represent the views of the National Aeronautics and Space Administration. 
Published by The RAND Corporation 
PRECEDING PAGE BLANK NOT FILMED.

-iii-

\section{PREFACE}

Observed zonal or meridional shifts of the geomagnetic field at the earth's surface suggest that similar motions may occur at the surface of the central core about half-way to the earth's center, assuming that the field lines are frozen into the surface of the core. The change of the earth's magnetic field in time is used to estimate possible motions within the core boundary.

The study presented here is one of a series intended to improve predictions of the strength and patterns of the earth's magnetic field as it affects the radiation belts, and to assist in estimates of the magnetic fields likely to be encountered on other planets.

This work was supported by the National Aeronautics and Space Administration. 


\section{PRECEDING PAGE ELANK NOT FILMED.}

$-v-$

\section{ABSTRACT}

The contribution to secular change made by the nonuniform velocity of the fluid at the surface of the earth's core in the presence of the earth's main field is discussed. It is shown that contributions to secular change made by temporal changes in the flow pattern of the fluid may be important. Results are discussed qualitatively in relation to dynamo theories of the earth's main field. It is also shown that surface fluid motions that can very nearly reproduce secular change also show features compatible with the generator models of Bullard, Herzenberg, Parker, and Tverskoy. The degree of compatibility found does not allow a preferred choice among these various generators. 
PRECEDING PAGE BLANK NOT FILMED.

-vii-

CONTENTS

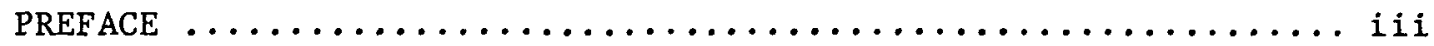

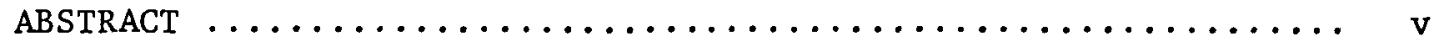

Section

I. INTRODUCTION $\ldots \ldots \ldots \ldots \ldots \ldots \ldots \ldots \ldots \ldots \ldots \ldots \ldots \ldots \ldots \ldots \ldots$

II. FLOW EQUATIONS $\ldots \ldots \ldots \ldots \ldots \ldots \ldots \ldots \ldots \ldots \ldots \ldots \ldots \ldots \ldots$

III. FIELD CHANGES DUE TO SURFACE TRANSLATION AND DISTORTION

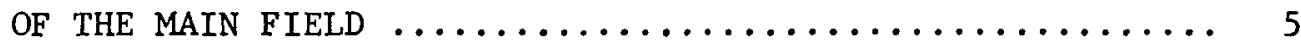

IV. CONTRIBUTIONS TO SECULAR CHANGE OF SURFACE VELOCITIES

WITH AND WITHOUT CURL $\ldots \ldots \ldots \ldots \ldots \ldots \ldots \ldots \ldots \ldots \ldots \ldots \ldots$

V. THE SURFACE FLOW IN RELATION TO DYNAMO THEORIES $\ldots \ldots \ldots \ldots .18$

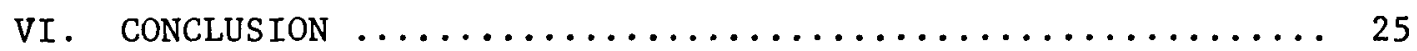

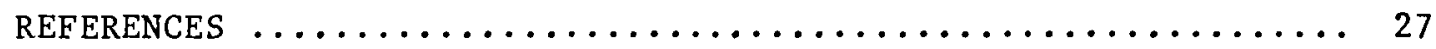




\section{INTRODUCTION}

The present paper extends our previous discussion, in a series of papers, of fluid motions at the surface of the earth's core (Vestine and Kahle, 1966; Kahle, Vestine, and Ba11, 1967; Kahle, Ba11, and Vestine, 1967). In this series we have continued to improve our approximations and have extended the time coverage of our estimates of the tangential velocities at the surface of the core that are necessary to produce the observed magnetic secular change. We have inferred the values of these velocities over the greater part of the past 100 years. The velocities were obtained by extrapolating the geomagnetic field and its secular change to the vicinity of the core, and then applying the frozen-field concept of geomagnetism (Alfvén and Fälthammer, 1963). Since the extrapolation greatly amplifies errors in the high-degree terms, we have limited our investigation to the broad-scale features of the velocity. Our theoretical approach in many respects parallels that of Roberts and Scott (1965), from whose work we have benefited great1y.

Such inferred data on surface velocities of the core are of special interest in connection with hydromagnetic theories of the origin of the geomagnetic field and its secular change (Elsasser, 1946a, b; Bullard, 1949; Alfvén and Fälthammer, 1963; Bullard, et a1., 1950; Bullard and Gellman, 1954; Runcorn, 1956; Parker, 1955; Allan and Bullard, 1958; Herzenberg, 1958; Nagata and Rikitake, 1961; Hide and Roberts, 1961; Lowes and Wilkinson, 1963; Hide, 1966). Assuming that all secular change can be regarded as arising primarily from tangential transport and distortion of the main poloidal field frozen in the fluid at the surface of the core, we show here that in approximately 80 percent of the grid points 
sampled on this surface, frozen transport of the field is more effective in generating secular change than is the accompanying distortion. We next show that the contributions to secular change at the earth's surface arising from (1) that part of the flow at the core surface that produces westward drift, (2) the remaining rotational flow, and (3) the irrotational flow, are comparable in magnitude. However, the westward drift considered separately makes a somewhat greater contribution to secular change. The poloidal terms in velocity at the surface of the core, suggested by Bullard and Gellman (1954), are substantiated, though they do not appear to be dominant features of the flow pattern. There is also a possibility that the rotational part of the flow may indicate the presence of two Herzenberg generators, one of which may serve to amplify the dipole field and the other to deamplify it. It is suggested that these generators may also provide a mechanism for the reversal of the main field. 


\section{FLOW EQUATIONS}

According to Elsasser (1946b), the velocity $\underline{v}$ within the core satisfies the equation

$$
\frac{\partial \underline{B}}{\partial t}=\nabla \times(\underline{v} \times \underline{B})-\frac{1}{4 \pi \sigma} \nabla^{2} \underline{B}
$$

where $\sigma$ is the electric conductivity and $\underline{B}$ is the magnetic field.

The last term, a diffusion term for the field $\underline{B}$, is generally assumed to be small when the magnetic field patterns are fairly simple (Elsasser, 1946b; Roberts and Scott, 1965; Kahle, Vestine, and Ba11, 1967). This assumption will be discussed more critically below and in a subsequent paper, but accepting it for the moment, one obtains

$$
\frac{\partial \underline{B}}{\partial t}=\nabla \times(\underline{v} \times \underline{B})
$$

Since $\nabla \cdot \underline{B}=0$, and $\nabla \cdot \underline{v}=0$ if the fluid is incompressible, we may rewrite Eq. (2) using a vector identity in the form

$$
\frac{\partial \underline{B}}{\partial t}=(\underline{B} \cdot \nabla) \underline{v}-(\underline{v} \cdot \nabla) \underline{B}
$$

Using polar coordinates $r, \theta, \lambda$ at the surface of the core, the radial component may be expressed in the form (Roberts and Scott, 1965; Kahle, Vestine, and Ball, 1967)

$$
\frac{\partial B_{r}}{\partial t}=-B_{r}\left(\frac{1}{r} \frac{\partial v_{\theta}}{\partial \theta}+\frac{v_{\theta} \cot \theta}{r}+\frac{1}{r \sin \theta} \frac{\partial v_{\lambda}}{\partial \lambda}\right)-\frac{v_{\theta}}{r} \frac{\partial B_{r}}{\partial \theta}-\frac{v_{\lambda} v_{\lambda}}{r \sin \theta} \frac{\partial B_{\lambda}}{\partial \lambda}
$$

For physical reasons, the horizontal-flow component of $\underline{\mathrm{v}}$ is more convenient1y expressed as the rotational and irrotational parts of $\underline{v}$ by finding the solution of Eq. (4) in the case where 


$$
\underline{v}=\underline{\mathrm{r}} \times \nabla x-\nabla \psi
$$

where

$$
\begin{aligned}
& x=\sum_{n=1}^{4} \sum_{m=0}^{n}\left(A_{n}^{m} \cos m \lambda+B_{n}^{m} \sin m \lambda\right) P_{n}^{m}(\theta) \\
& \psi=\sum_{n=1}^{4} \sum_{m=0}^{n} r\left(\alpha_{n}^{m} \cos m \lambda+\beta_{n}^{m} \sin m \lambda\right) P_{n}^{m}(\theta)
\end{aligned}
$$

and $\mathrm{r}$ is the radius of a spherical core (Kahle, Vestine, and Ball, 1967). In this expression the direction and magnitude of the rotational velocity along flow lines closing on the surface of the core are indicated by $\underline{\underline{r}} \times \nabla X$, and for irrotational flow, by $-\nabla \psi$.

The functions $X$ and $\psi$ in Eq. (5) may be related to the poloidal and toroidal vector flow-fields $\underline{S}$ and $\underline{T}$, respectively, by writing ${ }_{C}=-\partial \psi / \partial r,{ }_{C}=-X$, so that in Chandrasekhar's (1961) notation subscripting his variables with $C$,

$$
\begin{aligned}
& \underline{\underline{T}}=\nabla \times\left(\frac{\underline{\Psi}_{C}}{r} \underline{r}\right)=-\underline{r} \times \frac{\nabla_{\Psi} C}{r} \\
& \underline{s}=\nabla \times\left(\nabla \times \frac{\Phi}{r} \underline{r}\right)=-\nabla \times\left(\underline{r} \times \nabla \frac{\Phi}{r}\right)
\end{aligned}
$$


III. FIELD CHANGES DUE TO SURFACE TRANSLATION AND DISTORTION OF THE MAIN FIELD

Since at the moment we are interested only in large-scale horizontal flow, we assume that spherical harmonic terms up to degree 6 will suffice for $\underline{B}$ and to degree 4 for $\partial \underline{B} / \partial t$ (Kahle, Vestine, and Ball, 1967).

Table 1 lists the values of $(\underline{B} \cdot \nabla) \underline{v}$ and $(\underline{v} \cdot \nabla) \underline{B}$ at a number of points, calculated at $\mathrm{r}=3471 \mathrm{~km}$, using the data of Hendricks and Cain (1966) for epoch 1960. Although the second term is dominant about 80 percent of the time, the first is also important and is occasionally larger than the second. This means, of course, that small-scale spatial changes in the flow pattern sometimes contribute more effectively to secular change than do spatial variations of $\underline{B}$, and Table 1 suggests that this occurs more often at high latitudes. It therefore appears that the approximation $\underline{\partial} \underline{B} / \partial t=-(\underline{v} \cdot \nabla) \underline{B}$ used in our first paper (Vestine and Kahle, 1966) neglected a term which, though it is usually smaller than $-(\underline{\mathrm{v}} \cdot \nabla) \underline{\mathrm{B}}$, is nevertheless large enough in some regions to cause an error in the approximation of more than 100 percent. Such regions are clearly those in which $|(\underline{B} \cdot \nabla) \underline{y}|>|(\underline{v} \cdot \nabla) \underline{B}|$ in Table 1 .

It would therefore appear that if we adopt the frozen-field concept of hydromagnetism, the secular change, as shown by the radial component of the field, is generated mainly by the tangential translation of patterns in the main field. In about 20 percent of the cases examined, the local change in the horizontal flow pattern of the conducting fluid contributes more strongly than does translation.

It is also possible that $(1 / 4 \pi \sigma) \nabla^{2} \underline{B}$ may not be negligible in comparison with $(\underline{B} \cdot \nabla) \underline{v}$ or $(\underline{v} \cdot \nabla) \underline{B}$, especially for flow patterns of small 


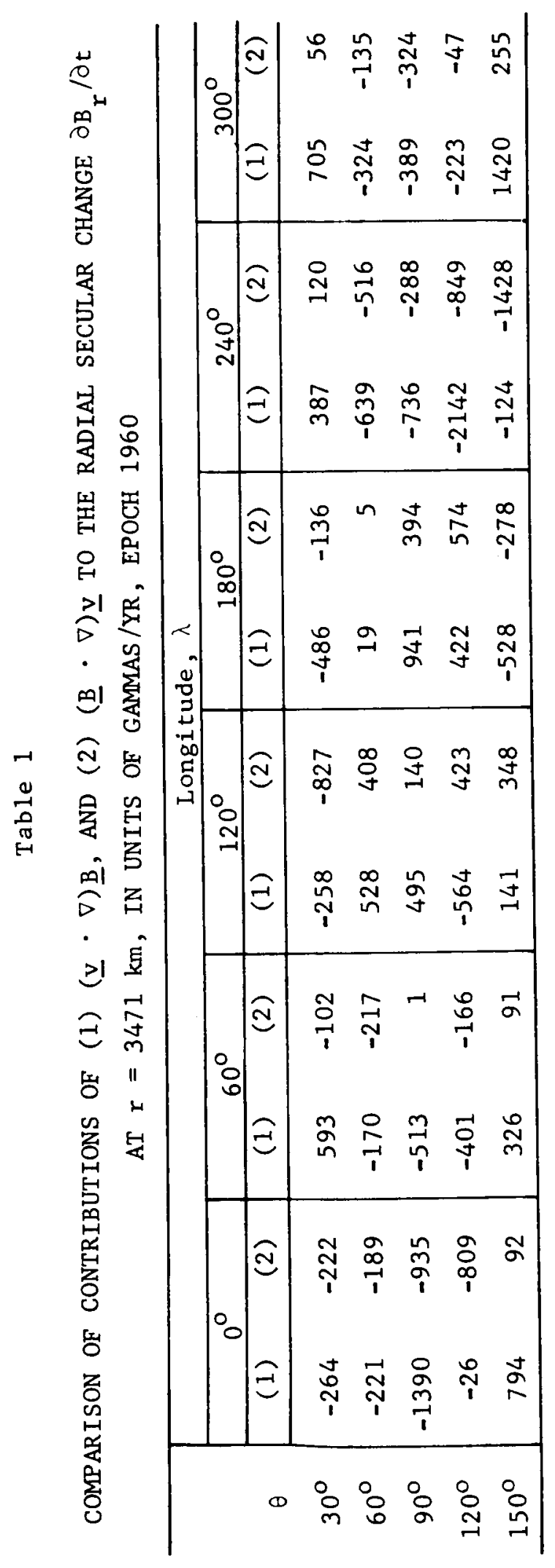


linear cross section (Kahle, Ball, and Vestine, 1967). We may also note that in general one cannot estimate $\nabla^{2} \underline{B}$, except when $\underline{B}$ is known to vary slowly with depth near the core surface, in which case $\nabla^{2} \underline{B}$ is negligible. We are indebted to Mr. Arthur Richmond (private communication) for pointing out that the flux $B_{r}$ of poloidal field lines entering the core along a radius may undergo rapid redistribution in density of field flux per unit area of core surface in the presence of a horizontal gradient $\partial \mathrm{B}_{\mathrm{r}} / \partial \mathrm{s}$, when distorted by a zonal flow of fluid. Since the magnitude of the contribution of $\nabla^{2} \underline{B}$ to $\partial \underline{B} / \partial t$ depends upon factors not available from the observational data, the effect of diffusion and lateral redistribution of the flux will not be considered here, even though it may exert some distorting influence on our flow patterns. We hope to return to this question in a future investigation. In the meantime, it may be noted that although the radial diffusion of $\underline{B}$ should be negligible in the case of the simple, broad-scale surface flow patterns considered here, a significant horizontal redistribution of the density of the radial flux lines would affect our estimates of $\underline{v}$.

As we have noted previously (Kahle, Bal1, and Vestine, 1967), a tangential flow parallel to isomagnetic lines of $\mathrm{B}_{\mathrm{r}}$ mapped on the surface of the core (Fig. 1) cannot be determined using Eq. (4), since this flow does not contribute to the secular change $\partial \underline{B} / \partial t$. The lines of force at the earth's surface will be carried along by the tangential flow, but the local flux $\underline{B}$ will remain unchanged there.

This nonuniqueness of the solution, corresponding to flows that preserve the magnetic flux density, was pointed out by Roberts and Scott (1965). The general form of these flows can be derived from Eq. (2) by 


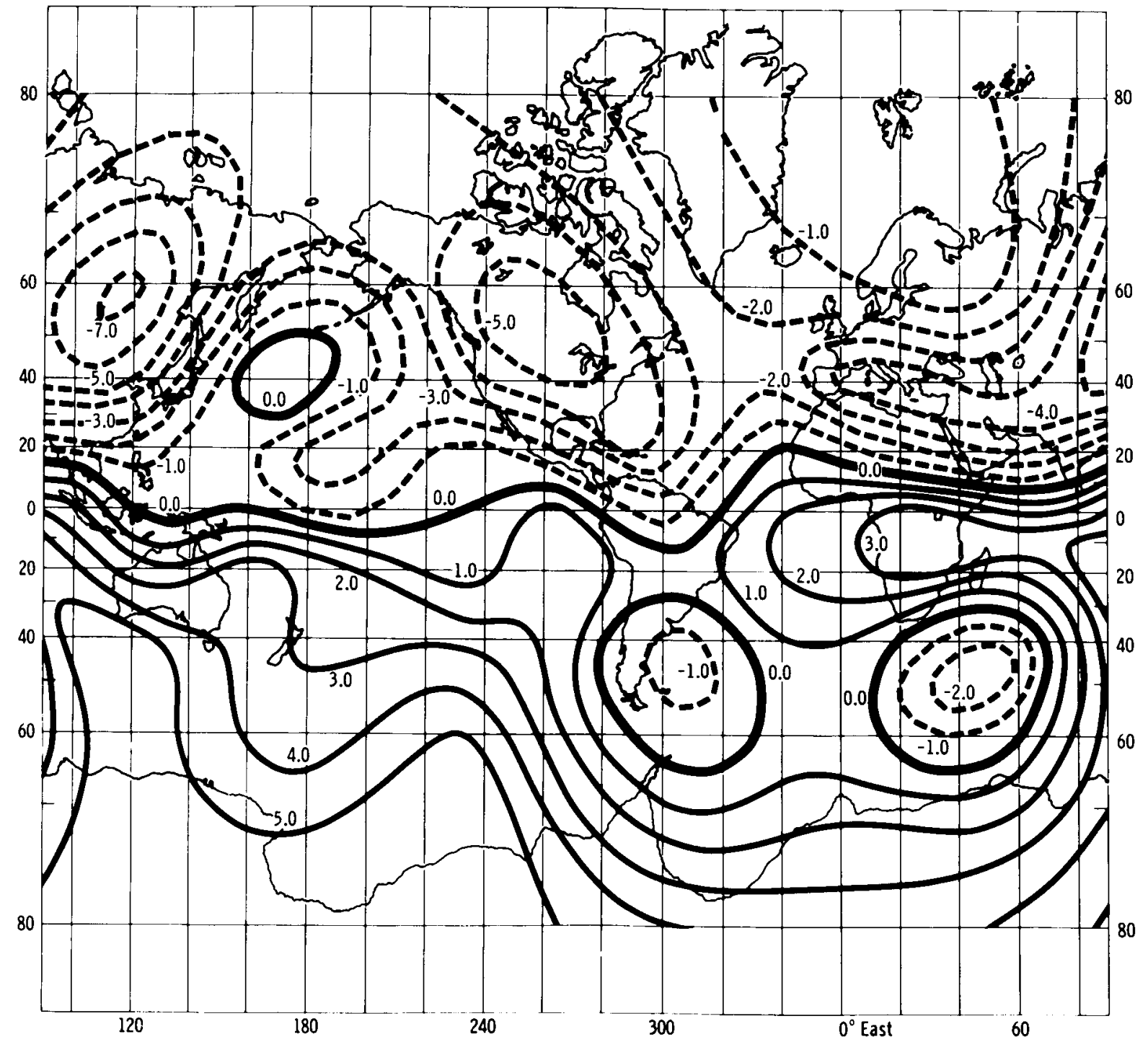

Fig. 1 -- Contours of the radial component, $B_{r}$, of the geomagnetic field at the surface of the core, $r=3471 \mathrm{~km}$, for epoch 1960. 
requiring that $\partial \underline{B} / \partial t=0$, which implies that the undetectable flow satisfies

$$
\underline{\mathrm{v}} \mathrm{x} \underline{\mathrm{B}}=\nabla \Phi
$$

for an arbitrary function $\Phi$ (essentially the electric potential). From the condition $y_{r}=0$ at the surface, one further obtains

$$
\mathrm{v}_{\mathrm{u}}=\frac{\mathrm{i}_{\mathrm{r}} \times \nabla \Phi}{\mathrm{B}_{\mathrm{r}}}
$$

where $\mathrm{v}_{\mathrm{u}}$ is the undefined part of the velocity.

If $\mathrm{v}_{\mathrm{u}}$ is to be nonsingular, $\Phi$ must vary like $\mathrm{B}_{\mathrm{r}}^{2}$, and of course $\Phi$ must be single-valued on the (closed) surface of the core. Without loss of generality, one can take $\Phi$ to be of the form

$$
\Phi(\theta, \lambda)=\left[\mathrm{B}_{\mathrm{r}}(\theta, \lambda)\right]^{2} \mathrm{f}(\theta, \lambda)
$$

so that

$$
\underline{\mathrm{v}}_{\mathrm{u}}=\mathrm{i}_{\mathrm{r}} \times\left[2 \mathrm{f} \nabla \mathrm{B}_{\mathrm{r}}+\mathrm{B}_{\mathrm{r}} \nabla \mathrm{f}\right]
$$

where $f$ is assumed to be continuous. The simplest example is $f=$ constant, which gives streamlines of $\mathrm{v}_{\mathrm{u}}$ identical with the contours of $\mathrm{B}_{\mathrm{r}}$ at the surface of the core, as shown in Fig. 1. Other permissible forms of $\mathrm{f}$ will generally give upflows and downflows as well as circulations, and these flows will always be more complicated than those of Fig. 1. In particular, Eq. (10) implies that the undetectable flow is always parallel to the contours of $\mathrm{B}_{r}$ in the vicinity of the contours $B_{r}=0$. It is therefore apparent that for a complicated $B_{r}$ pattern, the broad-scale features of the velocity pattern can be inferred fairly we11, whereas 
the smaller-scale patterns are subject to considerable uncertainty. Our knowledge of the smaller-scale velocity patterns could be improved by additional information, and some methods of acquiring this will be explored in a future article. 
IV. CONTRIBUTIONS TO SECULAR CHANGE OF SURFACE VELOCITIES WITH AND WITHOUT CURL

It is of some physical interest to consider the individual contributions to secular change of the parts of $\underline{v}$ with and without curl at the surface of the core. Table 2 lists a typical set of the harmonic coefficients of the velocity when the velocity at the surface of the core is assumed to be given by Eq. (5). The main magnetic field and $\partial \underline{B} / \partial t$ are those of the 1960 field as determined by Hendricks and Cain (1966). The westward drift is, by definition, the velocity obtained from Eq. (5) using only the terms $A_{n}^{0}$. The separate contributions of $\psi-A_{n}^{0}, X$ and westward drift to $\partial \underline{B} / \partial t$, shown in Table 3 , are obtained by using the coefficients of Table 2 in Eqs. (4) and (5). The sum of these contributions to $\partial \underline{B} / \partial t$ is given in Table 4 , along with the original $\partial \underline{B} / \partial t-$ field used, in terms of coefficients $\dot{g}_{n}^{m}$ and $\dot{h}_{n}^{m}$ at the earth's surface. On the whole, the agreement between computed and observed secular change at the earth's surface, harmonic by harmonic, is not particularly good. Some notable discrepancies appear, e.g. for the zonal component $\dot{\mathrm{g}}_{1}^{0}$, which has a computed value and an observed value of 5.6 and $20.6 \mathrm{y} / \mathrm{yr}$, respectively. However, when synthesized, the two fields appear basically quite similar. Figure 2 a shows the radial component of the secularchange field at the core, calculated from Hendricks and Cain's coefficients. Figure $2 b$ shows the same component synthesized from the coefficients in Table 4, which were derived from the velocity. Although the derived secular-change field appears considerably rougher, which is a natural consequence of combining several fields, the two fields agree much better than might be expected from comparing the coefficients of Table 4. 
Table 2

COEFFICIENTS OF VELOCITY AT THE SURFACE OF THE CORE IN KM/YR FOR $\underline{v}=\underline{r} \times \nabla X-\nabla \psi$, EPOCH 1960

\begin{tabular}{rr|rr|rr}
\hline & & \multicolumn{3}{|c|}{$\psi$} & \multicolumn{2}{c}{$\mathrm{X}$} \\
\cline { 3 - 6 } $\mathrm{n}$ & $\mathrm{m}$ & \multicolumn{1}{|c|}{$\alpha_{\mathrm{n}}^{\mathrm{m}}$} & \multicolumn{1}{c}{$\beta_{\mathrm{n}}^{\mathrm{m}}$} & \multicolumn{1}{c}{$\mathrm{A}_{\mathrm{n}}^{\mathrm{m}}$} & \multicolumn{1}{c}{$\mathrm{B}_{\mathrm{n}}^{\mathrm{m}}$} \\
\hline 1 & 0 & -1.42 & 0.00 & 3.53 & 0.00 \\
1 & 1 & 1.07 & 0.54 & 1.07 & -0.54 \\
2 & 0 & 0.12 & 0.00 & -1.59 & 0.00 \\
2 & 1 & -0.44 & 0.18 & 1.22 & 0.20 \\
2 & 2 & 0.21 & 0.33 & 0.09 & -0.40 \\
3 & 0 & -0.11 & 0.00 & -0.42 & 0.00 \\
3 & 1 & -0.16 & -0.23 & -0.78 & -0.58 \\
3 & 2 & -0.17 & -0.54 & 0.24 & 0.27 \\
3 & 3 & 0.32 & 0.67 & 0.55 & 0.34 \\
4 & 0 & -0.07 & 0.00 & 0.58 & 0.00 \\
4 & 1 & 0.06 & -0.08 & -0.07 & 0.46 \\
4 & 2 & -0.04 & 0.22 & -0.53 & -0.41 \\
4 & 3 & -0.19 & -0.12 & 0.13 & -0.80 \\
4 & 4 & 0.13 & 0.05 & 0.68 & -0.47 \\
\hline & & & & &
\end{tabular}


Table 3

CALCULATED SPHER ICAL-HARMONIC COEFFICIENTS OF SECULAR CHANGE AT THE EARTH'S SURFACE, DUE TO ROTATIONAL AND IRROTATIONAL PARTS OF THE VELOCITY FIELD, EPOCH 1960, UNITS OF GAMMAS/YR

\begin{tabular}{|c|c|c|c|c|c|c|c|}
\hline \multirow[b]{3}{*}{$\mathrm{n}$} & \multirow[b]{3}{*}{$\mathrm{m}$} & \multicolumn{4}{|c|}{ Rotational Field } & \multicolumn{2}{|c|}{ Irrotational Field } \\
\hline & & \multicolumn{2}{|c|}{$\begin{array}{c}\text { Westward } \\
\text { Drift }\end{array}$} & \multicolumn{2}{|c|}{ Remainder } & \multirow[b]{2}{*}{$\dot{g}_{n}^{m}$} & \multirow[b]{2}{*}{$h_{n}^{m}$} \\
\hline & & $\dot{\mathrm{g}}_{\mathrm{n}}^{\mathrm{m}}$ & $\mathrm{h}_{\mathrm{n}}^{\mathrm{m}}$ & $\dot{\mathrm{g}}_{\mathrm{n}}^{\mathrm{m}}$ & $\mathfrak{h}_{\mathrm{n}}^{\mathrm{m}}$ & & \\
\hline \multirow[t]{2}{*}{1} & 0 & - & -- & 2.4 & -- & 3.2 & -- \\
\hline & 1 & 9.2 & 2.2 & -3.5 & -14.2 & $-5 \cdot 4$ & 3.4 \\
\hline \multirow[t]{3}{*}{2} & 0 & -- & -- & -3.6 & $-\infty$ & -12.6 & -- \\
\hline & 1 & -3.0 & -4.7 & 0.4 & -4.1 & 6.2 & 0.7 \\
\hline & 2 & 0.6 & -0.3 & -2.5 & -4.7 & -1.1 & -4.8 \\
\hline \multirow[t]{4}{*}{3} & 0 & - & -- & 4.8 & -- & -1.3 & -- \\
\hline & 1 & -0.4 & 2.5 & -1.7 & 3.8 & -1.9 & -1.3 \\
\hline & 2 & 1.1 & -0.1 & 1.9 & -2.1 & -0.4 & 3.1 \\
\hline & 3 & 0.0 & -3.4 & 0.8 & -3.2 & -0.9 & -0.8 \\
\hline \multirow[t]{5}{*}{4} & 0 & - & -- & -2.1 & - & -0.2 & - \\
\hline & 1 & 0.5 & -0.4 & 1.7 & 1.0 & -2.6 & -1.3 \\
\hline & 2 & -0.9 & 0.2 & 0.5 & 1.6 & 0.5 & -1.9 \\
\hline & 3 & 0.4 & 1.3 & -1.0 & -0.4 & 0.2 & 1.8 \\
\hline & 4 & -0.6 & -2.0 & -1.5 & -2.3 & 0.1 & -0.2 \\
\hline
\end{tabular}


Table 4

COMPARISON OF SECULAR-CHANGE COEFFICIENTS COMPUTED FROM TOTAL DERIVED VELOCITY FIELD, WITH COEFFICIENTS OF THE OBSERVED SECULAR-CHANGE FIELD, EPOCH 1960, UNITS OF GAMMAS/YR

\begin{tabular}{|c|c|c|c|c|c|}
\hline \multirow[b]{2}{*}{$\mathrm{n}$} & \multirow[b]{2}{*}{$\mathrm{m}$} & \multicolumn{2}{|c|}{ Computed } & \multicolumn{2}{|c|}{ Observed } \\
\hline & & $g_{n}^{m}$ & $\mathrm{~h}_{\mathrm{n}}^{\mathrm{m}}$ & $\mathrm{g}_{\mathrm{n}}^{\mathrm{m}}$ & $\mathrm{h}_{\mathrm{n}}^{\mathrm{m}}$ \\
\hline \multirow[t]{2}{*}{1} & 0 & 5.6 & -- & 20.6 & - \\
\hline & 1 & 0.3 & -8.6 & 6.0 & -3.9 \\
\hline \multirow[t]{3}{*}{2} & 0 & -16.2 & $-\infty$ & -29.1 & -- \\
\hline & 1 & 3.6 & -8.1 & 1.2 & -13.7 \\
\hline & 2 & -3.0 & -9.8 & -0.7 & -15.8 \\
\hline \multirow[t]{4}{*}{3} & 0 & 3.5 & -- & 2.7 & - \\
\hline & 1 & -4.0 & 5.0 & -10.0 & 6.5 \\
\hline & 2 & 2.6 & 0.9 & 1.6 & 2.9 \\
\hline & 3 & -0.1 & -7.4 & -1.3 & -9.2 \\
\hline \multirow[t]{5}{*}{4} & 0 & -2.3 & -- & -0.9 & -- \\
\hline & 1 & -0.4 & -0.7 & 1.9 & -1.8 \\
\hline & 2 & 0.1 & -0.1 & -1.2 & -1.5 \\
\hline & 3 & -0.4 & 2.7 & -0.5 & 3.2 \\
\hline & 4 & -2.0 & -4.5 & -4.2 & -5.5 \\
\hline
\end{tabular}




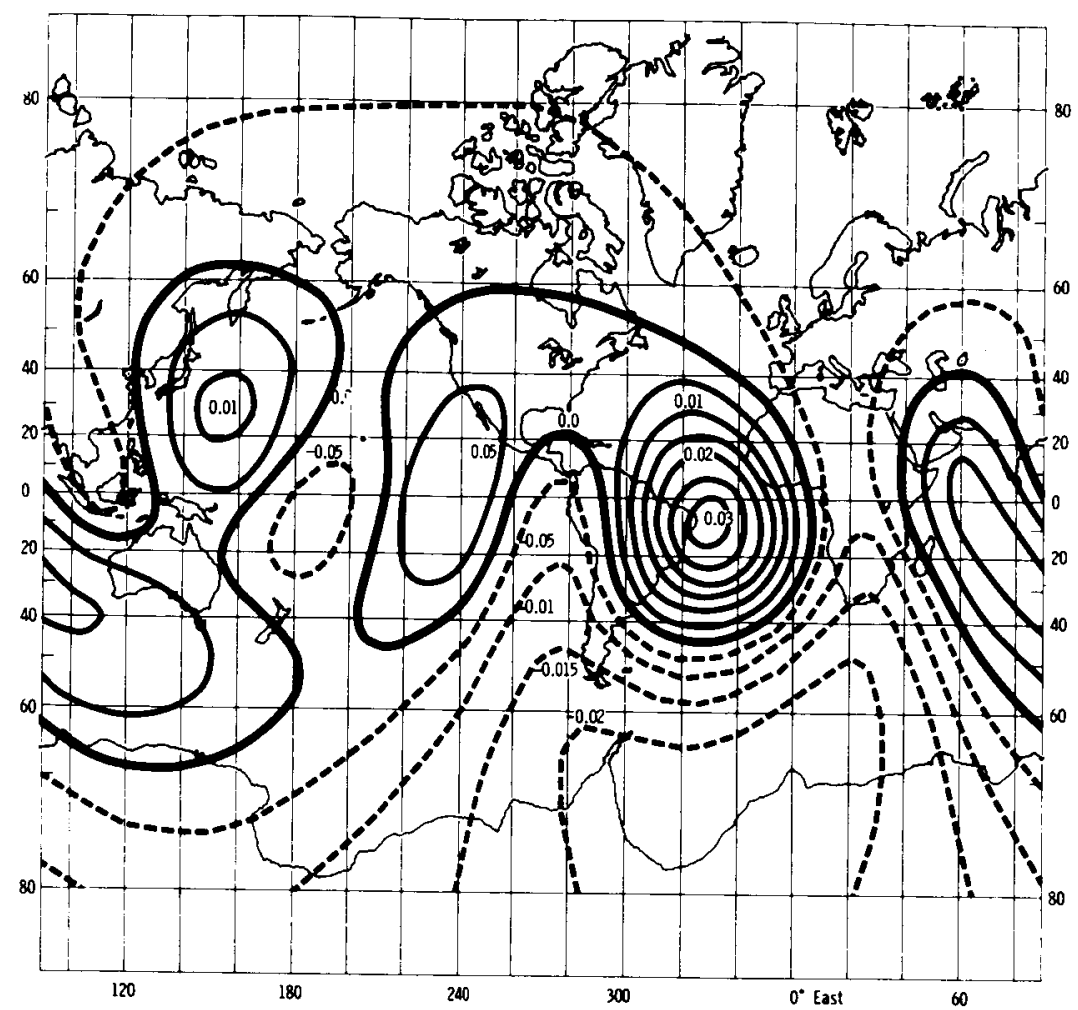

(a)

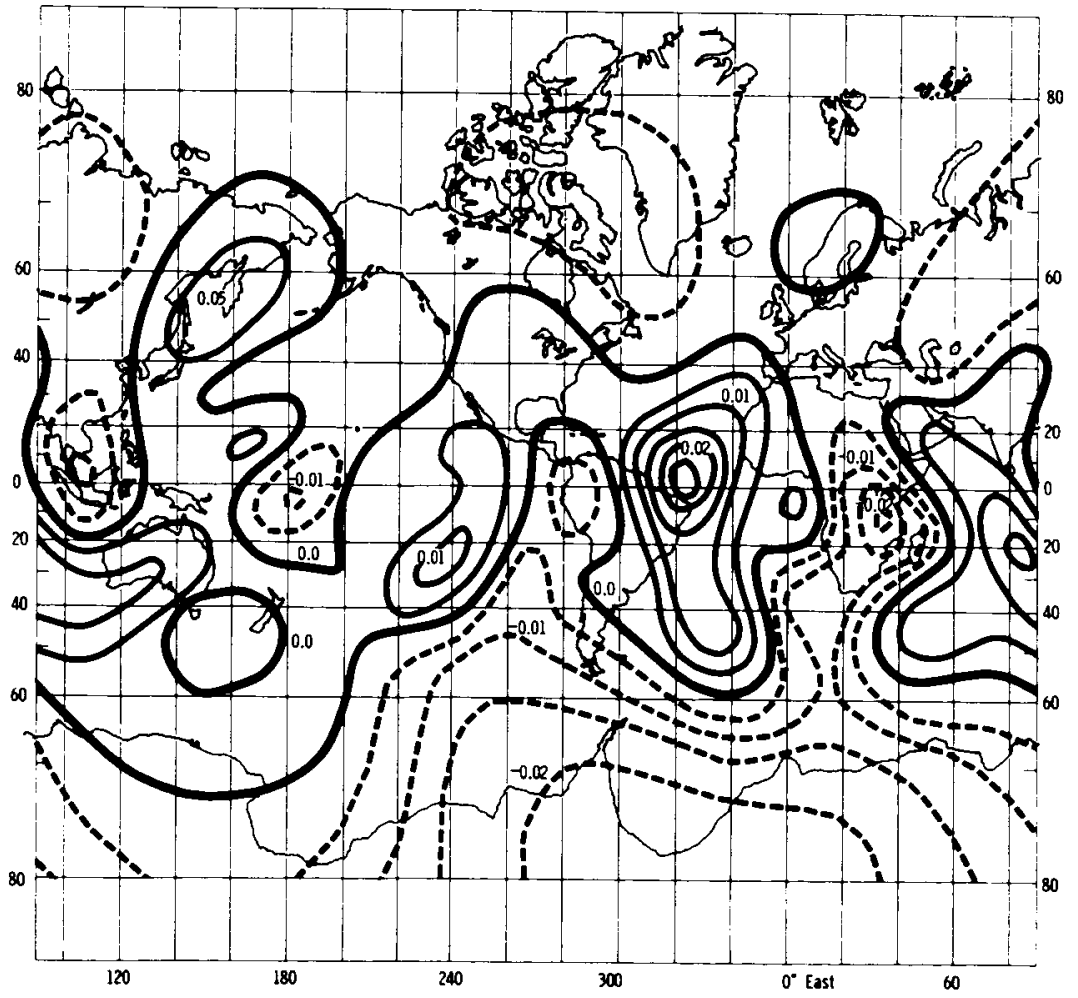

Fig. 2 - Radial component of secular-change field at the surface of the core, $r=3471 \mathrm{~km}$; (a) from secular-change coefficients of Hendricks and Cain, epoch 1960, (b) from calculated velocity. 
It may be noted that the transverse dipole terms $g_{1}^{1}, h_{1}^{1}$, for which current evidence shows at most only a very slight drift in any direction, apparently interact with the fluid motions of Table 2 to give some agreement with observation for secular change in the transverse dipole terms.

The secular-change coefficients of Table 3 are of course not directly comparable with the results of Yukutake (1962) and Nagata (1962), since they attributed secular change to terms in $A_{n}^{0} d_{n}^{0} / d \theta$. They assumed that the westward drift is $0.2 \% / y r$, and estimated the secular change which arises from westward drift of the nondipole field, rather than from this field plus the dipole field. The values of the remaining terms in $X$ and $\psi$, which make substantial contributions to $\partial \underline{B} / \partial t$, have to our knowledge not been estimated previously.

Nagata and Rikitake (1961) have investigated the interaction of poloidal fluid motions with toroidal fields in the core. An important term of the toroidal field presumably arises from interaction of the dipole term of the main field with the westward drift, giving a toroidal term $\mathrm{T}_{2}$. This term has a zonal field directed eastward in the core in middle latitudes of the northern hemisphere, and a similar but oppositely directed field in the southern hemisphere. Nagata and Rikitake found that the interaction of poloidal flows with the $\mathrm{T}_{2}$-field can generate a significant nondipole field. This field is generated slowly at first, and more rapidly after a thousand years or so. Consequently, motions such as those of Table 2, maintained for only a few hundred years, will not contribute much to the nondipole field as a consequence of their interaction with the $\mathrm{T}_{2}$-field. This result is, of course, in harmony 
with the assumptions used in estimating the fluid motions of Table 2 , in which the contribution of $\nabla^{2} \underline{B}$ was neglected. Nagata's (1962) estimates also indicate that the interaction of the motions in Table 2 with the poloidal field can generate a nondipole field quite rapidly - in only a few hundred years, on the basis of Eq. (5). The results of Table 3 support this view, since we note that nondipole components of the field can arise from the successive annual increments of the secular-change field during a century or so. It is not at all clear, however, whether the surface motions just inferred likewise predict the generation of the main dipole field. 


\section{THE SURFACE FLOW IN RELATION TO DYNAMO THEORIES}

We have previously found that in addition to the zonal flow or westward drift, a principal feature of fluid flow near the surface of the core is the downflow in the northern Pacific and the upflow near South Africa. Figures 3 and 4 exhibit graphically the flow patterns of Table 2 for 1960, exclusive of westward drift, which we recently estimated (Kahle, Ball, and Vestine, 1967) on the basis of the data of Hendricks and Cain (1966). The apparent westward zonal flow may indicate the occurrence of convection in the core (Bullard, et a1., 1950; Hide and Roberts, 1961). However, Hide (1966) has argued that the westward drift is due to propagation of hydromagnetic waves. The proposed explanations of the nonuniform rotation of the earth, however, give us reason to believe that the westward drift is nonuniform and rea1 (Kalinin, 1949; Munk and Revelle, 1952; Vestine, 1953; Hide and Roberts, 1961). For instance, the westward drift may have been less around 1910, coincident with a period when the crust and mantle rotated more rapidly (Vestine, 1962). These considerations lead us to believe that there is some zonal flow in the core.

We may also note that the substantial fluid motion of degree $n=2$ in Table 2 is of the type considered by Bullard and Ge11man (1954) in their numerical study of the dynamo theory. This term is

$$
\psi_{2}^{2}=r(0.21 \cos 2 \lambda+0.33 \sin 2 \lambda) \mathrm{p}_{2}^{2}(\theta)
$$

where $r$ is the radius of the core. The other second-degree term, $\psi_{2}^{1}$, is also fairly large. The $\psi_{2}^{2}$ term would provide a current feedback tending to amplify an original dipole field, as shown schematically in 


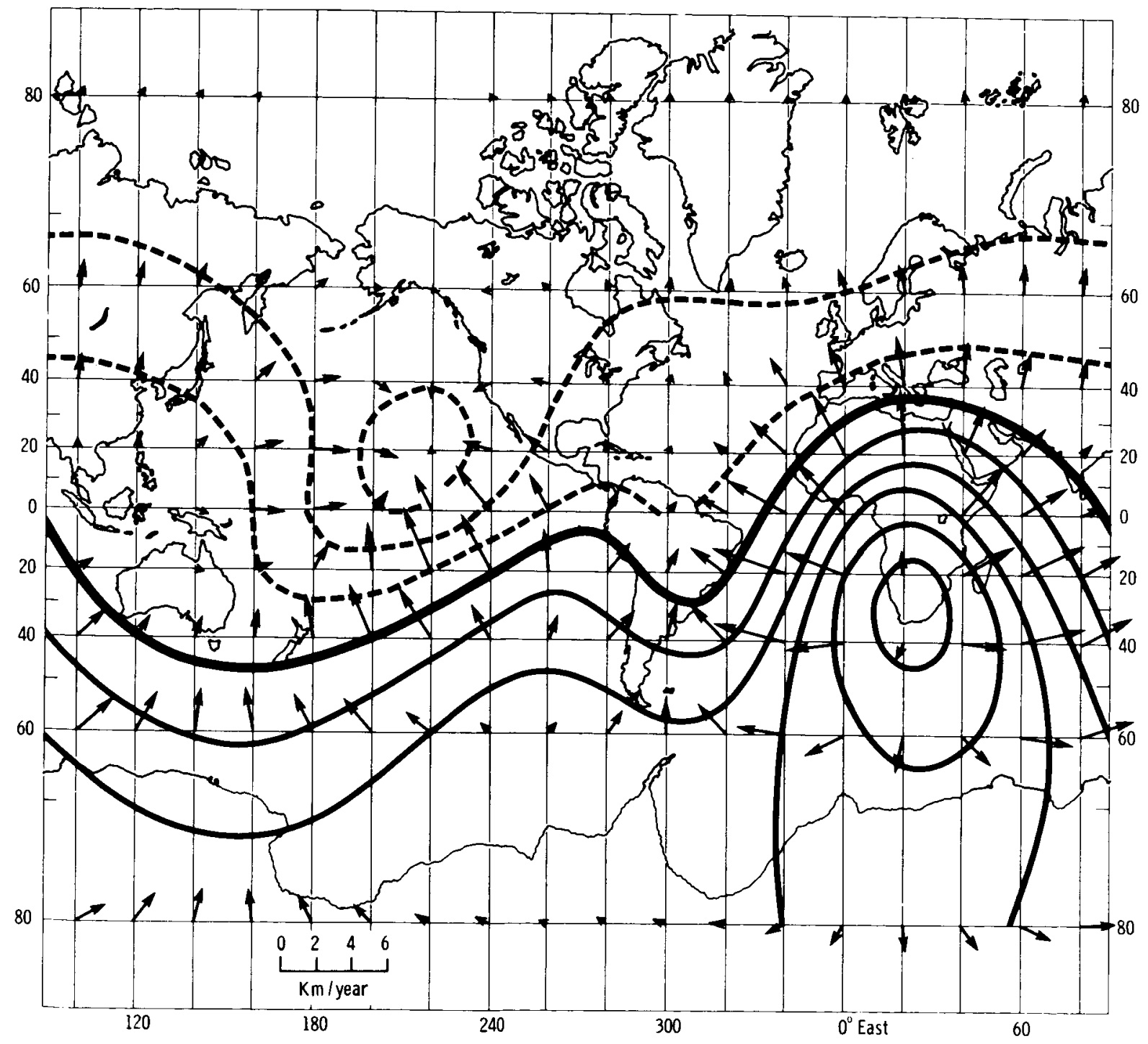

Eig. 3 -- Irrotational part of core surface velocity for epoch 1960. 


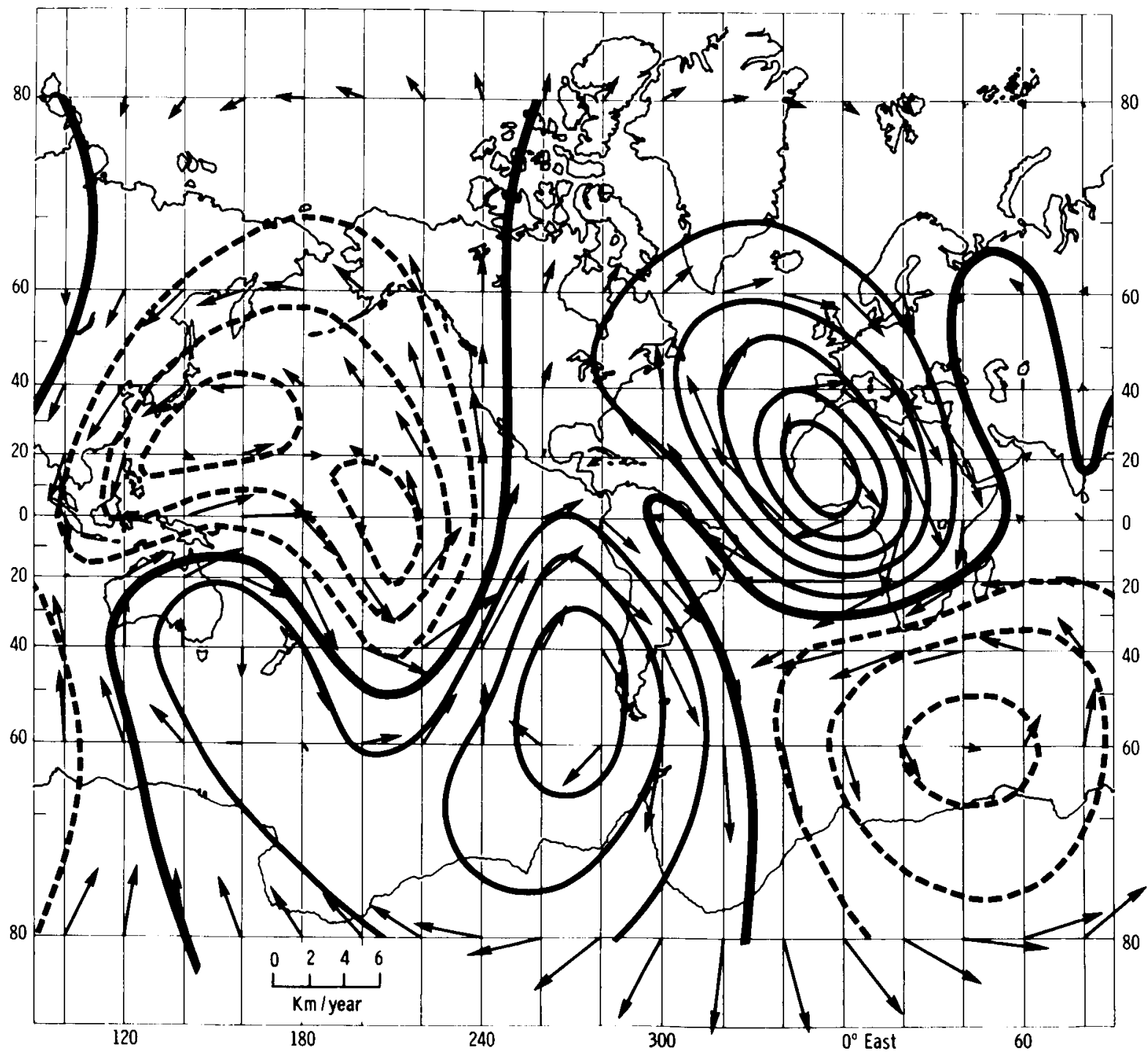

Fig. 4 -- Rotational part of core surface velocity minus the westward drift, for epoch 1960. 
Fig. 5. A grazing flow of the required type $\psi_{2}^{2}$ at the core surface has been deduced in our studies here, though inspection of Table 2 scarcely reflects a viewpoint that the term is especially dominant. Nevertheless, it is comparable in magnitude to the other terms $\psi_{n}^{m}$ (which we have suggested can, in any case, generate a nondipole field, probably in only a few hundred years).

It is of interest to mention that there may be evidence of a simple alternative dynamo for generating the main field. Figure 6 shows a type of generator considered by Herzenberg (1958), and also discussed recently by Lowes and Wilkinson (1963) and Rikitake and Hagiwara (1966). In this type of theory, two cylinders rotate in opposite directions inside a. conducting sphere; a dipole field can be generated if the cylinders are far enough apart. Lowes and Wilkinson built a working model of this type of dynamo. Though using solid rather than liquid cylinders.in rotation about axes approximately at right angles within a conducting fluid, they verified that it generated the predicted field. The data of Figs. 3 and 4 show that the flow which we deduced for grazing indidence at the mantle base appears to be of a type that they hoped might be present in the core of the earth, and barely visible in the flow at the top of the core. Whether our estimated flow persists to deeper levels is, of course, uncertain, but the simplest interpretation appears to imply that it does; moreover, the results indicate that a real dipole field may be generated on the basis of Herzenberg's dynamo.

Another simple generator of the geomagnetic field has been suggested by Tverskoy (1966). Unlike most theories of terrestrial dynamos, Tverskoy's is analytical. It postulates a vortex with meridional flow; 


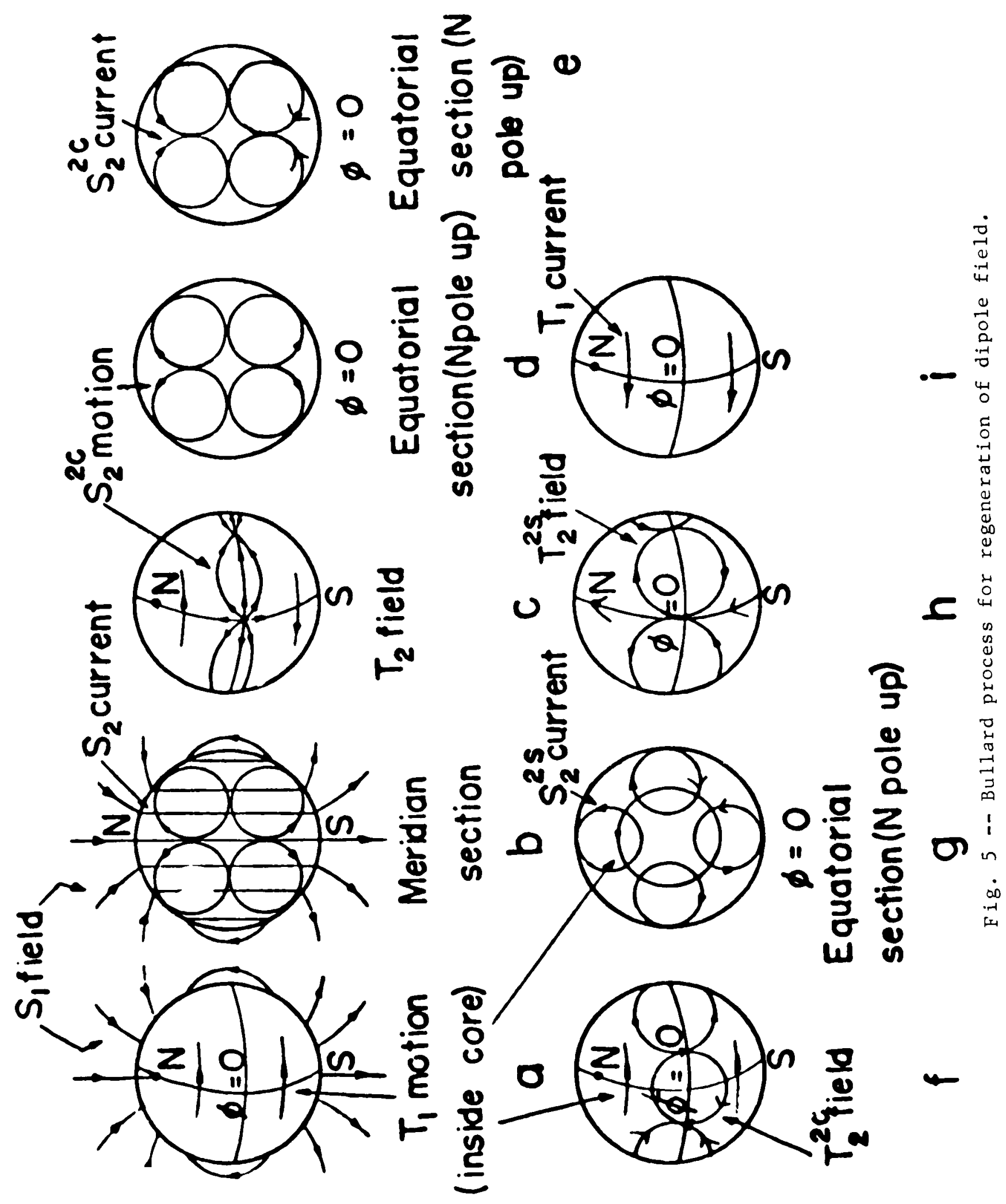


i.e., the vortex is symmetrical about the axis of rotation. According to Tverskoy, this vortex may generate a field that is initially nonaxial. Presumably the terms in $\mathrm{P}_{\mathrm{n}}^{0}$ of Table 2 are applicable here, since for $\mathrm{P}_{1}^{0}$ they yield a meridional flow of about $-1.42 \mathrm{~km} / \mathrm{yr}$ at the equatorial surface. A suitable poloidal-velocity function having a meridional flow of the form $P_{1}^{0}$ at the surface of the core and a vortex with meridional flow below is

$$
\Phi=\frac{b \alpha_{1}^{0}}{b-d}(r-b)(r-d) \cos \theta
$$

where $\alpha_{1}^{0}=-1.42 \mathrm{~km} / \mathrm{yr}$ is the surface value found in Table 2 , and $\mathrm{b}$ and $\mathrm{d}$ are the radii of the outer and inner (solid) core, respectively. The corresponding velocity is

$$
\begin{aligned}
\underline{v} & =-\nabla \times\left[\nabla \times\left(i_{r} \Phi\right)\right]=i_{r} \nabla_{T}^{2} \Phi-\nabla_{T}\left(\frac{\partial \Phi}{\partial r}\right) \\
& =\frac{b \alpha_{1}^{0}}{(b-d) r}\left[i_{\theta}(2 r-b-d) \sin \theta-2 i_{r} \frac{(r-b)(r-d)}{r} \cos \theta\right]
\end{aligned}
$$

which has been chosen so that $v_{r}$ vanishes on both the inner and outer surfaces of the core. The northward horizontal surface flow reverses to a southward flow for $r<(b+d) / 2$.

At the inner surface $(r \sim 1200 \mathrm{~km})$, the equatorial speed of meridional flow is $4.1 \mathrm{~km} / \mathrm{yr}$ in our example of a Tverskoy-type vortex generator; this speed was deduced in conformity with the meridional surface flow we have estimated for the outer core. According to Tverskoy, this type of flow, in the presence of an initial field, can generate a field which grows linearly with time, and with feedback of a suitable type can lead to a substantial generated field. 
The circulating eddies accompanied by an upflow of fluid in the Atlantic can also be discussed in relation to Parker's theory of the main field (1955). If a pulse of upflow pushes an east--west loop of the $\mathrm{T}_{2}$ field above the core surface, a rotation of the loop by $90^{\circ}$ in the senses shown for each eddy in Fig. 4 can yield a field-1ine loop emerging and descending at the core surface above each addy. These loops of field, expressed in spherical harmonics, would include a dipole contribution of the same sign as the original dipole field used to create $\mathrm{T}_{2}$. Since in this theory, downflow such as that in the Pacific would yield no poloidal term, the Pacific downflow need not be considered as a direct contributor to the dipole field.

Our purpose here has been to discuss in somewhat general terms only the more qualitative aspects of these generators. Other major aspects, which we have not yet discussed, include a more precise treatment of the boundary conditions at the core, as well as the contribution of diffusion to secular change. A more detailed and rigorous treatment of dynamo theories would be a matter of considerable computational difficulty, and seems presently infeasible (Bullard and Gellman, 1954; Stevenson and Wolfs on, 1966).

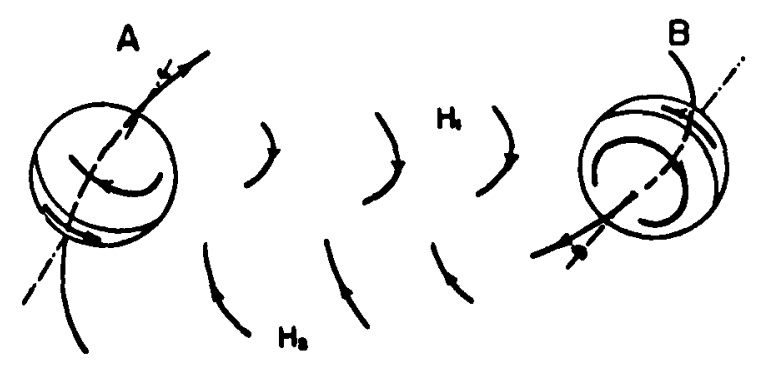

Fig. 6 -- Herzenberg dynamo. 


\section{CONCLUSION}

The surface flows tentatively estimated for the surface of the core, which show a dominant upflow near Africa and a downflow in the northern Pacific, can account for most of the secular change measured at the earth's surface and also for the nondipole field. These flows contain components which, on the basis of either Bullard's or Herzenberg's dynamo theory, may result in amplification or deamplification of the dipole field. The analytical results suggest the possible presence of two opposed Herzenberg generators, either of whose growth in time may cause a decrease, and eventual reversal, of the field. The rotational flow may also interact with toroidal fields to produce a feedback to the dipole term, leading to a pair of generators resembling those considered by Parker (1955). However, other types of generators, such as Tverskoy's, should also be considered. The estimates of surface flow in the core used in the present discussion of dynamos are, however, subject to possible revision, in the event that hydromagnetic waves and magnetic diffusion make a significant contribution to secular change. 


\section{REFERENCES}

Alfvén, H., and C. Fälthammer, Cosmical Electrodynamics, Clarendon Press, Oxford, 1963.

Allan, D. W., and E. C. Bullard, Distortion of a toroidal field by convection, Rev. Mod. Phys., 30, $1087-1088,1958$.

Bullard, E. C., The magnetic field within the earth, Proc. Roy. Soc. London, 197A, 433-453, 1949.

Bullard, E. C., C. Freedman, H. Gellman, and J. Nixon, The westward drift of the earth's magnetic field, Phil. Trans. Roy. Soc., 243A, $67-92,1950$.

Bullard, E. C., and H. Gellman, Homogeneous dynamos and terrestrial magnetism, Phi1. Trans. Roy. Soc., 247A, 213-278, 1954.

Chandrasekhar, S., Hydrodynamic and Hydromagnetic Stability, Clarendon Press, Oxford, 1961.

E1sasser, W. M., Induction effects in terrestrial magnetism, Part I, Theory, Phys. Rev., 69, 106-116, 1946a.

Elsasser, W. M., Induction effects in terrestrial magnetism, Part II, The secular variation, Phys. Rev., 70, 202-212, 1946b.

Hendricks, S. J., and J. C. Cain, Magnetic field data for trapped-particle evaluations, I. Geophys. Res., 71, 346-347, 1966.

Herzenberg, A., Geomagnetic dynamos, Phil. Trans. Roy. Soc. London, 250A, 543-585, 1958 .

Hide, R., Free hydromagnetic oscillations of the earth's core and the theory of the geomagnetic secular variation, Phil. Trans. Roy. Soc, London, 259A, 615-650, 1966. 
Hide, R., and P. H. Roberts, The origin of the main geomagnetic field, Physics and Chemistry of the Earth, 4, Pergamon Press, London, 27-98, 1961.

Kahle, Anne B., R. H. Ball, and E. H. Vestine, Comparison of Estimates of Fluid Motions at the Surface of the Earth's Core for Various Epochs, The RAND Corporation, RM-5193-NASA, March 1967.

Kahle, Anne B., E. H. Vestine, and R. H. Ball, Estimated surface motions of the earth's core, J.Geophys. Res., 72, 1095-1108, 1967.

Kalinen, Yu. D., Secular geomagnetic variations and changes in the length of the day, Meteorologiya i gidrologiya, No. 3, 15-19, 1949. Lowes. F. J., and I. Wilkinson, Geomagnetic dynamo: A laboratory model, Nature, 198, 1158-1160, 1963.

Munk, W., and R. Revelle, On the geophysical interpretation of irregularities in the rotation of the earth, Mon. Not. Roy. Astron. Soc., Geophys. Supp 1., 6, 331-347, 1952 .

Nagata, T., Two main aspects of geomagnetic secular varialion: Westward drift and non-drifting components, Proc. Benedum Earth Magnetism Symp., Pittsburgh, 39-55, 1962 .

Nagata, T., and T. Rikitake, Geomagnetic secular variation and poloidal magnetic fields produced by convectional motions in the earth's core, J. Geomag. Geoelec., 13, 42-53, 1961 .

Parker, E. N., Hydromagnetic dynamo mode1s, Astrophys. J., 122, 293-314, 1955.

Rikitake, T., and Y. Hagiwara, Non-steady state of a herzenberg dynamo, J.Geomag. Gevelec., 18, 393-409, 1966.

Roberts, P.H., and S. Scott, On analysis of the secular variation; (I) A hydromagnetic constraint: theory, J. Geomag. Geoelec., 17, 137-151, 1965. 
Runcorn, K., The present status of theories of the main geomagnetic field, Geologie en Mijbouw, 18, 347, 1956.

Stevenson, A. F., and S. J. Wolfson, Calculations on the 'Dynamo' problem of the earth's magnetic field, I. Geophys. Res., 71, 4446-4447, 1966.

Tverskoy, B. A., Theory of hydrodynamic self-excitation of regular magnetic fields, Geomag. Aeron., VI, 7-12, 1966.

Vestine, E. H., On variations of the geomagnetic field, fluid motions, and the rate of the earth's rotation, J.Geophys. Res., 58, 127-145, 1953.

Vestine, E. H., Influence of the earth's core upon the rate of the earth's rotation, Proc. Benedum Earth Magnetism Symp., Pittsburgh, 57-67, 1962 . Vestine, E. H., and Anne B. Kahle, On the small amplitude of magnetic secular change in the Pacific area, J. Geophys. Res., 71, 527-530, 1966. Yukatake, T., The westward drift of the magnetic field of the earth, Bu11. Earthquake Res. Inst., 40, 1-65, 1962. 


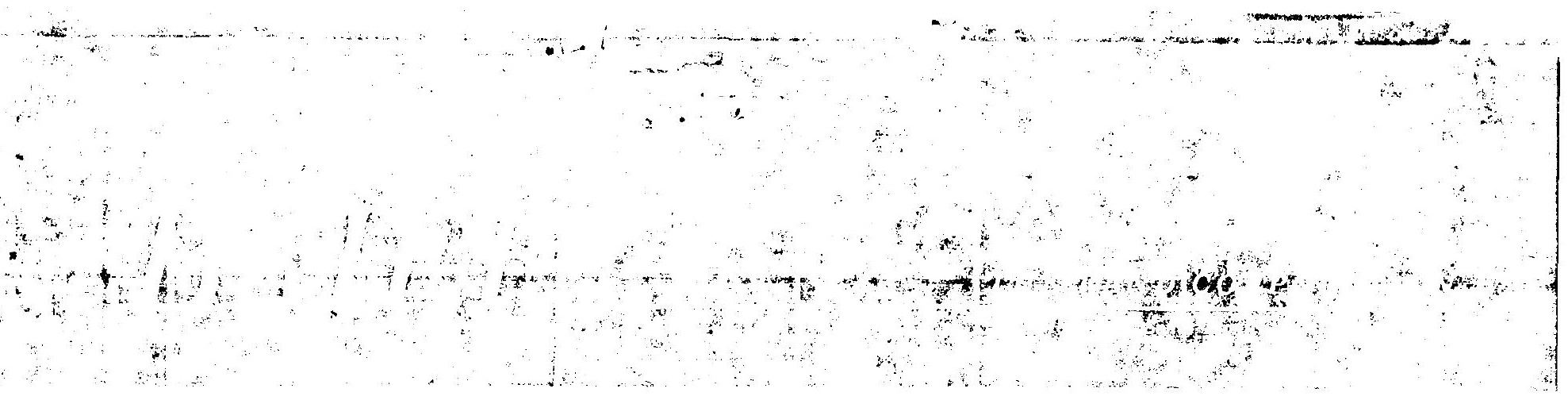

Apri 11967

RB-5 192

RM-5192-NASA, Nature of Surface Flow in the Earth's Central Core, E. H. Vestine, R. H. Ba11, and Anne B. Kahle, RAND Memorandum, April $1967,36 \mathrm{pp}$.

PURPOSE: To estimate velocities of the fluid at the surface of the earth's core. Data on these velocities are of special interest in connection with hydromagnetic theories of the origin of the geomagnetic field and its secular change.

RELATED TO: RAND's research for the National Aeronautics and Space Administration. This study is one of a series intended to improve predictions of the strength and patterns of the earth's magnetic field as it affects the radiation belts. The series is planned to assist in estimating magnetic fields likely to be encountered on other planets. Previously published studies include RM-5193-NASA, Comparison of Estimates of Surface Fluid Motions of the Earth's Core for Various Epochs, April 1967; RM-5191-NASA, Westward Drift of the Geomagnetic Fie1d and Its Relation to Motions of the Earth's Core, December 1966; and RM-5091-NASA, Estimated Surface Motions of the Earth's Core, August 1966.

METHODOLOGY AND DISCUSSION: Observed zonal or meridional shifts of the geomagnetic field at the earth's surface suggest that similar motions may occur at the surface of the central core about halfway to the earth's center, assuming that the field lines are frozen into the surface of the core. The change of the earth's magnetic field in time is used to estimate possible motions within the core boundary.

The tangential velocities of the fluid at the surface of the earth's core were obtained by extrapolating the geomagnetic field and its secular change to the vicinity of the core, and then applying the frozen-field concept of geomagnetism. Since the extrapolation greatly amplifies errors in the high-degree terms, the investigation is limited to the broad-scale features of the velocity.

FINDINGS: The surface flows tentatively estimated for the surface of the core, which shows a dominant upflow near Africa and a downflow in the Northern Pacific, can account for most of the secular change measured at the earth's surface and also for the nondipole field. These flows contain components that, on the basis of the dynamo theory of either Bullard (1954) or Herzenberg (1958), may result in amplification or deamplification of the dipole field. The analytical results suggest the possible presence of two opposed Herzenberg generators, either of whose growth in time may cause a decrease, and eventual reversal of the field. The rotational flow may also interact with toroidal fields to produce a feedback to the dipole term, leading to a pair of generators resembling those considered by Parker (1955). However, other types of generators, such as that of Tverskoy (1966), should also be considered. The estimates of surface flow in the core used in the present discussion of dynamos are, however, subject to possible revision in the event that hydromagnetic waves or magnetic diffusion makes a significant contribution to secular change. 